\title{
Evaluation of different statistical shape models for segmentation of the left ventricular endocardium from magnetic resonance images
}

\author{
Concetta Piazzese ${ }^{1,2}$, M. Chiara Carminati ${ }^{3}$, Andrea Colombo ${ }^{1}$, Rolf Krause $^{2}$, Mark Potse ${ }^{2}$, Lynn \\ Weinert ${ }^{4}$, Gloria Tamborini ${ }^{3}$, Mauro Pepi ${ }^{3}$, Roberto M. Lang ${ }^{4}$, Enrico G. Caiani ${ }^{1}$ \\ ${ }^{1}$ Dipartimento di Elettronica, Informazione e Bioingegneria, Politecnico di Milano, Italy \\ ${ }^{2}$ Università della Svizzera Italiana, Lugano, Switzerland \\ ${ }^{3}$ Centro Cardiologico Monzino IRCCS, Milan, Italy \\ ${ }^{4}$ Department of Cardiology, University of Chicago, IL, USA
}

\begin{abstract}
Statistical shape models (SSMs) represent a powerful tool used in patient-specific modeling to segment medical images because they incorporate a-priori knowledge that guide the model during deformation. Our aim was to evaluate segmentation accuracy in terms of left ventricular $(L V)$ volumes obtained using four different SSMs versus manual gold standard tracing on cardiac magnetic resonance $(C M R)$ images. A database of $3 D$ echocardiographic (3DE) LV surfaces obtained in 435 patients was used to generate four different SSMs, based on cardiac phase selection. Each model was scaled and deformed to detect $L V$ endocardial contours in the enddiastolic (ED) and end-systolic (ES) frames of a CMR short-axis (SAX) stack for 15 patients with normal LV function. Linear correlation and Bland-Altman analyses versus gold-standard showed in all cases high correlation $\left(r^{2}>0.95\right)$, non-significant biases and narrow limits of agreement.
\end{abstract}

\section{Introduction}

Cardiac magnetic resonance (CMR) imaging is nowadays one of the preferred non-invasive imaging modalities to study the left ventricle (LV). In fact, the non-invasive nature and its ability to modulate contrast in response to several mechanisms or to provide highquality functional and anatomical information in any plane and any direction constitute important advantages of CMR imaging compared to other modalities [1].

Quantification of global and regional LV function in terms of stroke volume, ejection fraction, wall thickness, and wall thickening depends upon accurate delineation of the endocardial wall. However, manual tracing of each 2D short-axis (SAX) slice in the acquired stack is time- consuming, tedious, and characterized by inter-observer variability $[2,3]$.

Moreover, when the analysis is aiming to generate 3D meshes for patient-specific electro-mechanical models, slice misalignment or low spatial resolution in-between slices could result in morphological artifacts and in a poor representation of the LV base and apex. Statistical shape modelling (SSM) has been proposed as a technique, based on some a-priori knowledge (i.e. shape or intensity), able to generate accurate segmentations of the LV. Heimann et al. [4] described various statistical models and their biomedical application. We recently proposed a method to segment the LV endocardium in CMR SAX stack based on a SSM built on a database of 3D endocardial meshes segmented from transthoracic 3D echocardiographic (3DE) images [5].

The aim of this work was to compare the segmentation accuracy of the LV endocardium on CMR images by using four different SSMs, built on different frame selections from the available database.

\section{Methods}

\subsection{Shape Model Generation}

Transthoracic 3DE datasets (iE33, Philips) of 435 retrospective patients (293 with normal LV function, 78 with dilated cardiomyopathy, 13 with aortic insufficiency, 11 with aortic stenosis, 28 with mitral regurgitation and 12 with mitral stenosis) evaluated at the University of Chicago, IL, USA or at Centro Cardiologico Monzino, Italy, formed the database. For each frame in the cardiac cycle, the LV surface was obtained as a 3D mesh (TomTec), for a total of 7010 3D meshes in the database. Four different SSM were generated: 1) end-diastolic (ED) model, in which only meshes corresponding to ED frames were considered; 2) end-systolic (ES) model, in which 
only meshes corresponding to ES frames were considered; 3) ED-ES model, including only meshes corresponding to both ED and ES frames; 4) global model, including meshes from all frames.

For each SSM, the corresponding meshes were coregistered, and the mean LV endocardial surface was computed (Figure 1). To describe the variability of the point cloud, principal component analysis (PCA) was performed, with 1926 principal components (PC) to describe the whole data variability.

\subsection{Population and gold standard (GS)}

CMR ECG-triggered steady-state free precession SAX (slice thickness $8 \mathrm{~mm}$, no gap, no overlap) stacks acquired together with long-axis (LAX, two- and fourchamber planes orientation) views in 15 retrospectively selected patients with normal LV function were analyzed. ED and ES frames were visually selected, and an expert cardiologist traced the LV contours, from which the ED and ES volumes were computed and used as 'gold standard' (GS) for comparison.

These images were analysed using the proposed approach by separately applying each of the generated SSM to both the ED and the ES frames. In addition, we tested also the strategy of applying the frame-specific model to each frame individually (i.e., for ED frames the ED model, and for ES frames the ES model). For each segmentation, also the number of iterations required by the deformation process to converge was considered.

\subsection{Pre-processing and user initialization}

As SSM segmentation works on the whole 3D stack, to minimize potential breathing artefacts the method proposed by Carminati et al. [6] to correct for spatial misalignment was applied. A cross-correlation technique performed an in-plane correction in each SAX plane, using LAX views as reference. Furthermore, to reduce noise and preserve edges, all images were smoothed used a bilateral filter (filter half-width $=9 \mathrm{~mm}, \mathrm{SD}$ in coordinate space $=9 \mathrm{~mm}, \mathrm{SD}$ in intensity space $=0.05$ ) [7]. In order to properly scale and position each SSM inside the CMR data to be analysed, six points (two for mitral valve leaflet insertion and one for LV apex, in LAX views) were manually initialized by the user.

\subsection{Image segmentation and model deformation}

The segmentation method takes as input each scaled SSM and iteratively deforms the model until a stable condition is reached. At each iteration, the intersections between each SAX plane and the SSM are computed (Figure 2a) along with the neighborhood points that lie on the line connecting each intersection with the LV centre of mass (Figure 2b) in a circular region of interest (20 pixels for apical SAX planes, 60 pixels for basal SAX planes).

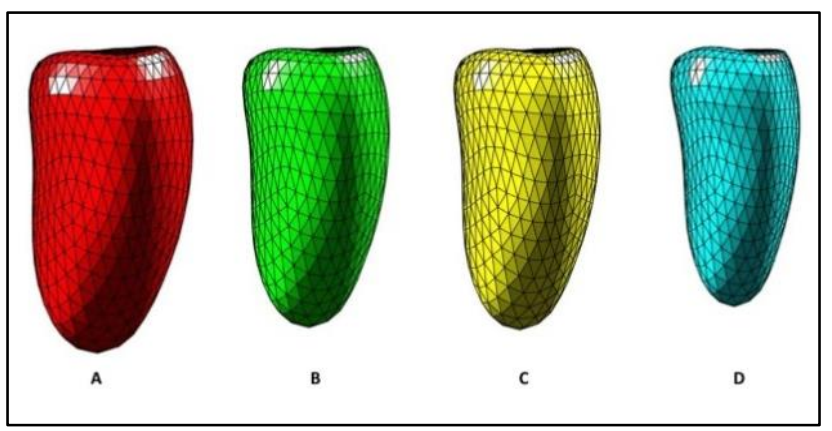

Figure 1. Mean shape of the four SSMs, from larger to smaller resulting LV endocardial surface : (A) ED model; (B) Global model; (C) ED-ES model; (D) ES model.

Then, for each SAX plane, a radial image is obtained by polar-to-Cartesian coordinates transformation applied to each videointensity line profile. Depending on the position of each SAX plane, a k-means clustering with different number of clusters (from 3 for apical SAX planes to 5 for basal SAX planes) followed by binarization was applied to the radial image, thus resulting in white areas corresponding to the LV cavity and black areas corresponding to the myocardium and external structures. The interface pixels were then extracted (Figure 2c) and transformed back to Cartesian coordinates (Figure 2d) on the SAX plane. In order to include papillary muscle in the LV cavity, each set of interface points was analysed to find the smallest convex set that contains it. Pixel positions detected at the border of the convex hull were used as the LV endocardial candidate nodes in the iterative update process of the SSM (Figure 2e). Once all SAX planes were similarly analysed, Procrustes analysis [8] was applied to perform a rigid repositioning of the SSM model in the actual SAX stack, and for each node a displacement vector based on the position of the respective candidate points was computed. After the rigid registration, the shape of each 3D SSM was globally deformed to match the LV endocardial position in all SAX planes simultaneously. Model deformation ability was restricted in the range $(-3 \sigma \div+3 \sigma)$ of the PC that statistically describe the points cloud from which the corresponding SSM was generated.

Once model update was ended, LV volume was computed as the sum of volumes of all the regular tetrahedral patches composing the 3D mesh (3D). 

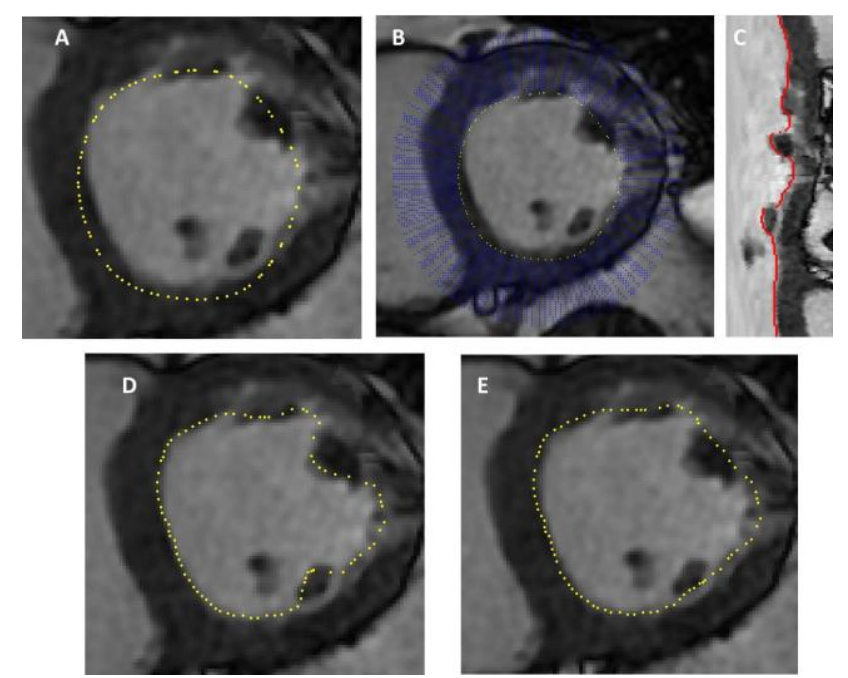

Figure 2. Different steps of the SAX segmentation process. (A) Intersection points (yellow) between the initial mean SSM and one short-axis image; (B) Lines connecting each intersection points to the centre of mass (blue, only a segment was traced for simpler visualization); (C) Radial image, created by transforming line videointensity profiles from Cartesian to polar coordinates, with superimposed the segmented endocardial edge (red); (D) Corresponding endocardial edge (yellow) pixels in Cartesian coordinates; $(E)$ Contour points (yellow) after the application of a convex hull filter in order to include papillary muscles.

\subsection{Statistical analysis}

Semi-automated volumes corresponding to ED and ES frames in each patient were computed using each SSM model, and compared to the manual GS volumes using linear correlation and Bland-Altman analysis. Furthermore, since volumes obtained are neither independent nor fit a normal distribution, a nonparametric statistical method (Friedman analysis) with multiple comparisons was applied in order to test differences between endocardial SSM volumes.

\section{Results}

The segmentation algorithm was implemented in Matlab (MathWorks). LV endocardial segmentation and volume computation using SSMs was possible in all 15 patients. Convergence of the fitting process required around 1 minute for each CMR stack of images, using a conventional laptop (Intel_Core i5 @ $2.5 \mathrm{GHz}, 8 \mathrm{~GB}$ RAM). Manual tracing of the whole SAX stack for GS determination took about $5 \mathrm{~min}$ per frame.

Figure 3 shows an example of the final SSM superimposed to LAX views. This visualization highlights the correspondence of the detected surface, deformed by a stack of SAX contours, to the LV endocardial position in LAX planes, and its correspondence with the LV apex and base.

Correlations between the GS and the SSM measured volumes were significant and similar in all four cases (Figure 4, left), with slopes close to 1 and high correlation coefficients $\left(r^{2}>.95\right)$. Bland-Altman analysis revealed for all SSMs no significant biases compared with GS volumes and relatively narrow limits of agreement (Figure 4, right), slightly wider when the ED model was used. Friedman's test showed that none of the explored segmentation strategies resulted in different LV volumes than the GS. Iterations to convergence were very similar for each strategy, with a median value of 5 and interquartile difference of 0 . Again, using the ED model interquartile difference increased to 1 .

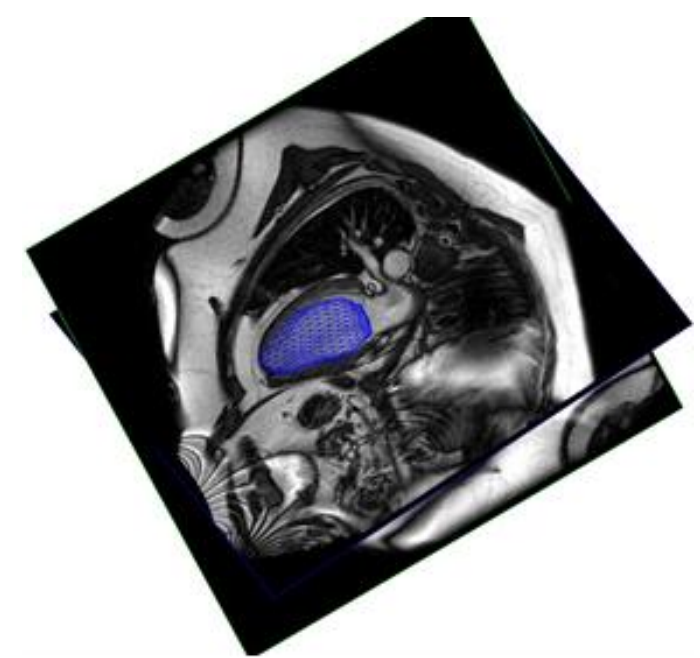

Figure 3. Final ED model (blue) superimposed on LAX views. The shape of the model is deformed based on short-axis segmented contours until a stable condition is reached.

\section{Discussion}

This study aimed at comparing segmentation performances based on utilization of four different SSMs to detect the LV endocardium from SAX CMR images, using manual tracing as the reference standard. All models were built starting from the same database of $3 \mathrm{DE}$ LV surface meshes, but including a different selection of frames from each patient, according to the SSM.

To our knowledge, this is the first study aimed at comparing SSMs built from 3DE surfaces and then applied to segment CMR images. In our preliminary study [5], a single SSM model built using all frames in the cardiac cycle was applied, and a smaller 3DE database was used. 

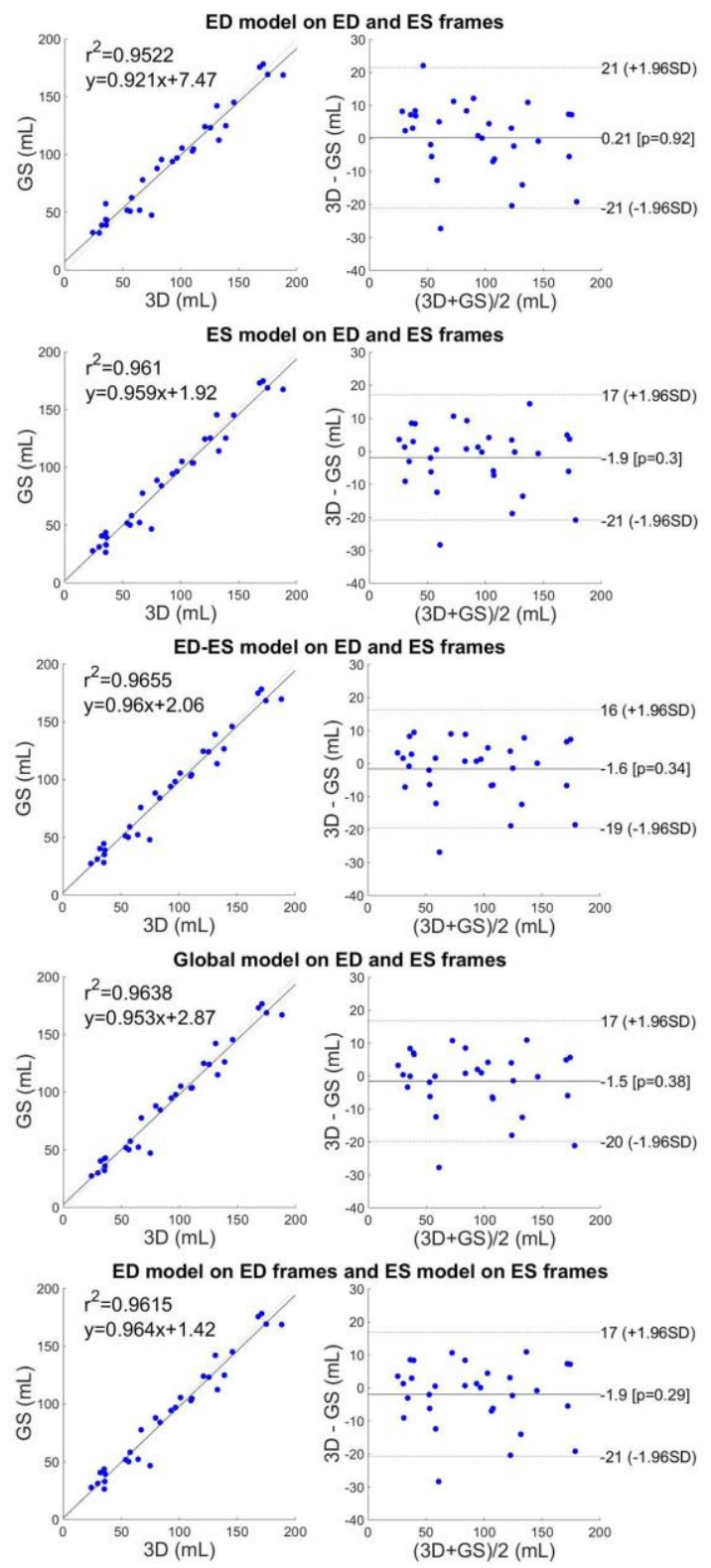

Figure 4. Linear correlation and Bland-Altman analyses between the 'gold standard' manually derived LV volumes and the $3 D$ volumes obtained using different SSM segmentation strategies.

The comparisons of the explored strategies of segmentation with the LV volumes obtained by conventional manual GS showed a very good agreement in all cases. Independently of the starting SSM, the segmentation process was able to accurately define the endocardial wall even if the resulting 3D shape was constrained in the range of variability defined by the PCA components of the corresponding SSM. This ability could be due to the large number of subjects included in the database, covering a large morphological variability.
Slightly worst results were observed using the ED model, in terms of confidence interval and number of required iterations to convergence.

\section{Conclusion}

We evaluated the accuracy of four different SSMs to segment the LV endocardium using a novel intermodality statistical modelling approach. The utilization of 3D LV endocardial surfaces extracted from 3DE images as training datasets allowed generating different SSMs that all resulted in good agreement with LV volumes obtained by manual tracing.

\section{Acknowledgements}

This work was supported by the Italian Space Agency (contract number 2013-032-R.0, recepient E.G.C.).

\section{References}

[1] Pattynama PMT, De Roos A, Van der Wall EE, Van Voorthuisen AE. Evaluation of cardiac function with magnetic resonance imaging. Am Heart J 1994;128:595607.

[2] van Assen HC, Danilouchkine MG, Dirksen MS, Reiber JH, Lelieveldt BP. A 3-D active shape model driven by fuzzy inference: application to cardiac CT and MR. IEEE Trans Inf Technol Biomed. 2008 Sep;12(5):595-605..

[3] Lu Y, Radau P, Connelly K, Dick A, Wright G. Segmentation of left ventricle in cardiac cine MRI: An automatic image-driven method. Funct. Imag. Model. Heart, pp. 339-347, 2009.

[4] Heimann T, Meinzer HP. Statistical Shape Models for 3D Medical Image Segmentation: A Review. Medical Image Analysis, Vol. 13, No. 4, pp. 543-563, 2009..

[5] Caiani EG, Colombo A, Pepi M, Piazzese C, Maffessanti F, Lang RM, Carminati MC. 3D Left ventricular segmentation from MRI images for patient-specific modeling purposes. Europace 2014;16(suppl_4):iv96-101iv

[6] Carminati MC, Maffessanti F, Caiani EG. Nearly automated motion artifacts correction between multi breathhold short-axis and long-axis cine CMR images. Comput Biol Med 2014;46:42-50.

[7] Tsadok Y, Petrank Y, Sarvari S, Edvardsen T, Adam D. Automatic segmentation of cardiac MRI cines validated for long axis views. Computerized Medical Imaging and Graphics, Volume 37, Issues 7-8, October-December 2013, 500-511.

[8] Gower J. Generalized Procrustes analysis. Psychometrika 1975;40:33-51.

Address for correspondence:

Concetta Piazzese

Dipartimento di Elettronica, Informazione e Bioingegneria Politecnico di Milano, Piazza L. da Vinci 32, Milano, Italy E-mail: concetta.piazzese@polimi.it 